\title{
Control of distension of varicose veins achieved by leg bandages, as used after injection sclerotherapy
}

\author{
P H FENTEM， MARILYN GODDARD， B A GOODEN， C K YEUNG
}

British Medical fournal, 1976, 2, 725-727

\section{Summary}

A study was performed to determine whether the pressures routinely produced by bandaging for compression sclerotherapy of varicose veins are adequate to maintain the superficial veins almost empty of blood. The results suggest that well-applied bandages can provide sufficient support to combat the high distending pressures found in varicose veins. The large variation among different surgeons, however, indicates that any clinical assessment of compression sclerotherapy should include measurement of the pressure at which the bandages are applied.

\section{Introduction}

The success of injection sclerotherapy of varicose veins seems to depend on uninterrupted compression of the veins at the sites of injection. The objective is to produce a permanent fibrous occlusion of the veins at certain points to partially correct abnormal pressure gradients and flow patterns. ${ }^{1}$ Fegan has suggested that the best clinical results are obtained if the sclerosant is injected into an empty vein and the vein is then compressed for at least six weeks to enable fibrous occlusion of the vein to take place. In clinical practice, it is usual to provide compression by some form of bandaging, often with the inclusion of a rubber or polyether foam pad over the injected vein.

In a previous study Fentem et al ${ }^{2}$ showed that support garments which provide only modest compression can control the distension of varicose veins. This is possible because the relation between pressure and radius, as described by Laplace $(\mathbf{P}=\mathbf{T} / \mathbf{R})$, is such that the pressure exerted on a distended vein by a compression garment greatly exceeds the overall compression provided to the limb, the vein having a much smaller radius than the limb.

The same favourable relation will apply when bandages are used. This study was undertaken to determine whether bandaging, applied at tensions that do not cause discomfort to the patient or impair arterial inflow to the limb, is nevertheless adequate to combat the high distending pressures found in varicose veins and even to maintain the veins almost empty of blood. The benefit of including foam pads 'under the bandages was also assessed.

\section{Methods}

Nine healthy subjects were invited to take part in the first study. They were four men and five women of average build, with heights

\footnotetext{
Department of Physiology and Pharmacology, University Hospital and Medical School, Nottingham NG7 2UH

P H FENTEM, MSC, MB, CHB, professor of physiology

MARILYN GODDARD, BSC, research assistant

B A GOODEN, MD, lecturer in physiology

General Hospital, Nottingham NG1 6HA

C K YEUNG, MB, FRCS, registrar in surgery
}

ranging from 1.56 to $1.80 \mathrm{~m}$ and calf circumferences of $32-36 \mathrm{~cm}$. The right leg of each subject was bandaged by the same surgeon using one type of bandage in three ways: (a) two Crevic crepe bandages (width $10 \mathrm{~cm}$, length $4.5 \mathrm{~m}$ ) were applied from the foot to below the knee; $(b)$ two Crevic crepe bandages were applied over a narrow polyether foam pad of triangular cross-section (base $5 \mathrm{~cm}$, height $3 \mathrm{~cm}$, length $19 \mathrm{~cm}$ ); (c) two Crevic crepe bandages were applied over a broad polyether foam pad of trapezoidal cross-section (base $11 \mathrm{~cm}$, height $3 \mathrm{~cm}$, length $19 \mathrm{~cm}$ ).

The subject rested supine on a couch with the leg supported beneath the knee and heel by foam blocks to raise the calf from the couch. Simultaneous measurements were made of bandage compression applied around the circumference of the leg and the control of distension of a varicose vein model by the bandages.

The bandage compression was measured using a large, flat, polyvinylchloride bag $(100 \times 80 \mathrm{~mm})$ containing $5 \mathrm{ml}$ of water. ${ }^{2}$ The bag was connected by nylon tubing (internal diameter $1 \mathrm{~mm}$ ) to a Bell and Howell L221 pressure transducer and thence to an ultraviolet recording device (SE 3006). The bag was secured by its edges with adhesive tape to the posterior aspect of the calf, where it moulded readily to the leg contours, and covered a large area of the limb surface. The bandage was applied in the usual manner over the bag and a pressure reading was taken.

At the same time the control of distension of a varicose vein model by the bandages and compression pads was studied. The model, secured to the anterolateral aspect of the calf, was made from a $150-\mathrm{mm}$ length of Viskin tubing (volume $25 \mathrm{ml}$, diameter $15 \mathrm{~mm}$ ) sealed on to polyethylene tubing (internal diameter $2.2 \mathrm{~mm}$ ). The pressure within the model was measured and recorded as described above. Water was infused into the model at a rate of $4 \mathrm{ml} / \mathrm{min}$ by a syringe located between the model and the pressure transducer, using an infusion pump (Sage 351). A control pressure-volume curve was recorded. The foam pads were positioned directly over the long axis of the model vein, and extended $20 \mathrm{~mm}$ beyond the model at each end. The leg was bandaged and subsequent pressure-volume curves were obtained. Each subject was bandaged twice by each method, the order being randomised.

For each subject the distance between the fourth intercostal space in the anterior mid-line and mid-calf level was measured, and the theoretical hydrostatic pressure distending a vein at this level was calculated. We assumed that for an upright and motionless subject there was a continuous column of blood from the heart to calf level. From the control pressure-volume curve of the uncovered model vein, the volume contained in the model at the theoretical hydrostatic pressure was noted. The percentage volume reduction of the vein model at this distending pressure, achieved by the pads or bandages, or both, was calculated as follows:

$\%$ volume reduction $=100-\left\{\frac{\text { volume of model under bandage }}{\text { volume of model uncovered }} \times 100\right\}$ at hydrostatic pressure.

Bandaging pressures achieved by five surgeons-For the second part of the study five surgeons who were routinely engaged in treating varicose veins by compression sclerotherapy were invited to apply leg bandages to three men with similar leg proportions. Each surgeon, using two Crevic crepe bandages, bandaged the leg from the foot to below the knee 10 times. The bandage compression was measured as described above.

\section{Results}

The values for the compression achieved by Crevic crepe bandages applied using the three bandaging methods are shown in the table. The same surgeon bandaged 18 legs. The compression achieved when the limb was bandaged without a pad was significantly higher $(P<5 \%)$ than when either type of pad was included. There was no significant difference in bandage pressure with the narrow pad or the broad pad.

The extent to which a particular bandage limited the distension of the model vein was deduced from pressure-volume curves. Examples 
Overall compression and corresponding percentage volume reduction of vein model achieved by three bandaging methods. Results are means $\pm 1 S E$

\begin{tabular}{l|c|c}
\hline \multicolumn{1}{c|}{$\begin{array}{c}\text { Bandaging } \\
\text { method }\end{array}$} & $\begin{array}{c}\text { Compression } \\
\text { (mm Hg) }\end{array}$ & $\begin{array}{c}\text { \% Volume reduction } \\
\text { of vein model }\end{array}$ \\
\hline (a) Bandage alone & $54.3 \pm 2.2$ & $93.6 \pm 0.75$ \\
(b) Bandage and narrow pad & $46.9 \pm 2.5$ & $94.3 \pm 0.85$ \\
(c) Bandage and broad pad & $43.4 \pm 2.3$ & $89.0 \pm 1.22$ \\
\hline
\end{tabular}

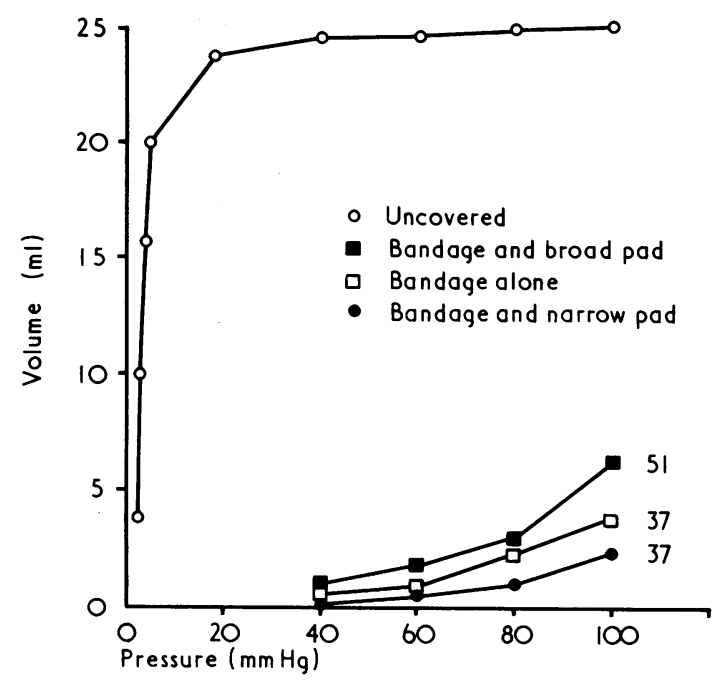

FIG 1-Pressure-volume relation of vein model when uncovered and under bandages. Bandage compression $(\mathrm{mm} \mathrm{Hg})$ is shown for each curve.

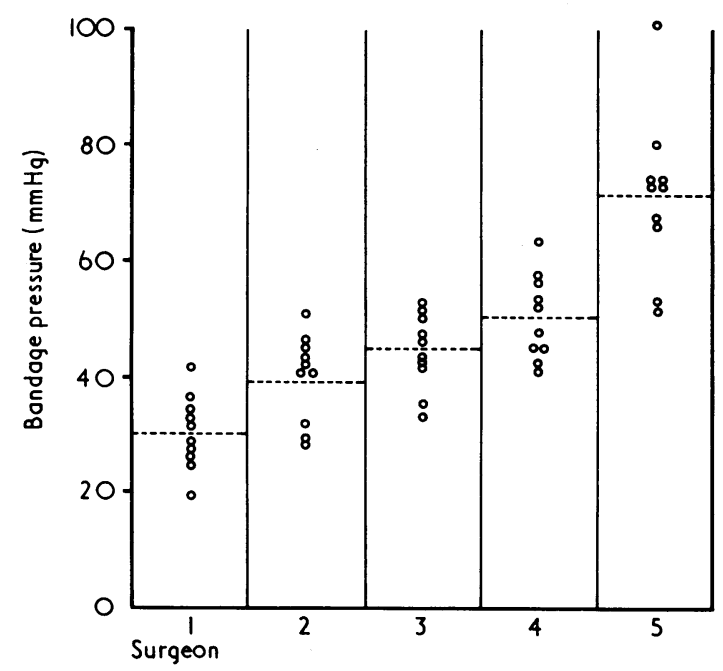

FIG 2-Bandage compression achieved by five surgeons. Each surgeon performed 10 bandagings. Horizontal line represents mean value.

of these curves recorded when the three methods were used on an individual leg are shown in fig 1 . With the bandage in place, the reduction in the capacity of the model vein was almost complete at distending pressures below $60 \mathrm{~mm} \mathrm{Hg}$ and was greater than $90 \%$ at $80 \mathrm{~mm} \mathrm{Hg}$ for all three methods. The compression achieved by the three methods varied since bandage tension could not be controlled.

Mean values for the reduction in the capacity of the model vein observed for each method of bandaging on 18 legs are presented in the table (right-hand column). Methods $a$ and $b$ both reduced distension by about $94 \%$. This reduction was achieved by a significantly lower bandage pressure when the narrow pad was included, however. The smallest reduction in distension was observed when a broad pad was used, although bandage pressure was not significantly lower than for method $b$.

Bandaging pressures achieved by five surgeons-The range of bandage pressures is shown in fig 2. The individual variation in bandage pressure achieved was much less than the overall range, which extended from $20 \mathrm{~mm} \mathrm{Hg}$ to a maximum of $100 \mathrm{~mm} \mathrm{Hg}$. Thus, although the differences between the three bandaging methods when used by one surgeon were significant, the range of bandage pressures achieved by different surgeons was much larger.

\section{Discussion}

The contributions made by different factors to the success of compression sclerotherapy have not been fully evaluated. The tension at which an individual surgeon applies his bandages is rarely known to him and is not a detail included in clinical studies where bandaging has been an important component of the treatment, which is the case with injection sclerotherapy. The consequences of including foam pads under the bandages do not seem to have been detrimental, but the effect of these pads on compression was unknown. The dimensions of the pad might also be critical. The compression exerted by bandages of different constructions has rarely been measured and requires further study.

The mean compression achieved using Crevic bandages in this study was $54 \mathrm{~mm} \mathrm{Hg}$ at calf level. The bandages, applied by CKY, were comfortable. A varicose vein model distended to the maximum hydrostatic pressure for the subject (ranging from 70 to $90 \mathrm{~mm} \mathrm{Hg}$ ) had a volume only $6 \%$ of that when it was not covered by bandages (see table). The same reduction in capacity of the model vein was achieved by a bandage pressure of only $47 \mathrm{~mm} \mathrm{Hg}$ when a narrow pad was placed over the model before bandaging. The use of the narrow pad has advantages in improved comfort for the patient since bandages may be:applied at a reduced tension without a reduction in the compression over the injected vein. The inclusion of the broad pad reduced the local pressure over the vein model, and thus a narrow pad is better.

The greater success of the narrow pad in compressing the varicose vein model may be partly explained by the fact that the effective radius of the bandage over the apex of the narrow pad is much less than the average radius of the calf. The Laplace relation would predict a higher local pressure where the bandage crossed the top of the pad $(P=T / R)$. Conversely, the broad pad has a larger, flattened surface, which produces an effective radius greater than that of the leg, and thus the local pressure under the pad is less for the same bandage tension. The mean overall bandage pressure was lower when either pad was included. Since the pads increase the overall circumference of the calf, and thus increase its mean radius, the bandage pressure is probably reduced.

The behaviour of the model vein is likely to differ from that of a real vein in several respects. It stands above the skin surface and may have more support from the underlying tissues than a real vein. Experiments were carried out to determine the effect of structure of the vein model on pressure-volume relations, and results obtained using segments of long saphenous varicose veins were very similar to those of the model. The effect of model diameter was also considered, and these investigations are described elsewhere. ${ }^{3}$

This method of comparing the local pressures applied to the model vein by bandages alone with those applied when a foam pad is included under the bandage may well underestimate the benefit provided by a narrow foam pad. Whereas the bandage may fail to empty a vein lying partially supported by subcutaneous tissue as successfully as it empties a model vein on the surface of the leg, a narrow pad may make it possible for bandaging to completely compress the vein even when it lies in a gutter between tissues. The narrow pad will tend to sink into the soft tissue under the pressure of the bandage.

The variation in bandage pressures achieved by the five surgeons was large. Nevertheless, surgeons 2, 3, and 4, when bandaging according to no specific instructions, achieved mean pressures of 40,45 , and $50 \mathrm{~mm} \mathrm{Hg}$ respectively. Examination of individual pressure-volume curves suggested that these 
pressures would reduce the capacity of a superficial varicose vein, distended to the maximum hydrostatic pressure, by more than $90^{\circ}{ }_{0}$. Surgeon 1 achieved a mean bandage pressure of $30 \mathrm{~mm} \mathrm{Hg}$, which would reduce the capacity of the vein model by only $75^{\circ}$ o if no pads were included. It is still unresolved, however, whether it is more important to consider the volume of the compressed vein at the hydrostatic distending pressure or at mean venous ambulatory pressures, which will be lower if the calf muscle pump is efficient. The hydrostatic pressure is the highest sustained pressure likely to be experienced by the vein, though very high local pressure transients may occur at the junction of an incompetent perforating vein and a varicose vein when the calf muscles contract forcefully. These high pressures may not generally last long enough to reopen a partially sclerosed segment of vein under compression. Venous ambulatory pressures of $52 \mathrm{~mm} \mathrm{Hg}$ have been recorded from superficial foot veins in patients with venous insufficiency. ${ }^{4}$ When the vein model is distended to this lower pressure the same bandage compression of only $30 \mathrm{~mm} \mathrm{Hg}$ would reduce the capacity of the model by $96^{\circ}$.

The optimum bandage pressure for compression sclerotherapy has still to be defined, although our results may suggest an effective range of pressures. In a few experiments pressurevolume curves were recorded from the vein model placed under a National Health lightweight support stocking, which exerted a pressure of $8.5 \mathrm{~mm} \mathrm{Hg}$ around the calf. This pressure reduced the capacity of the model by only $22^{\circ}{ }_{0}$ and is clearly inadequate for sclerotherapy treatment. A bandage pressure greater than $50 \mathrm{~mm} \mathrm{Hg}$, however, is said to be high enough to represent a potential hazard to the arterial inflow to the limb under certain circumstances. ${ }^{5}$ Results from this study suggest that when a narrow foam pad is included over the injection site pressures as high as $50 \mathrm{~mm} \mathrm{Hg}$ are not necessary, assuming that successful fibrous occlusion of the vein can take place if its capacity is reduced by at least $90 \%$ at maximum hydrostatic distending pressures. Although it is not known how empty the injected vein must be during the treatment, more complete emptying of the vein may reduce the incidence of complications such as thrombophlebitis associated with sclerosant injections. For this reason, bandage pressures of more than $30 \mathrm{~mm} \mathrm{Hg}$ are possibly indicated, since below this pressure a significant volume of blood may be present within the vein when it is subjected to high distending pressures.

The considerations discussed in this paper point to the need to measure the pressure applied by limb bandages when assessing the treatment of varicose veins by compression sclerotherapy. Clearly the degree of compression achieved is more variable than many would have believed. Individual surgeons should be aware of the pressures at which they apply compression bandages. The scope of our study did not make it possible to determine whether these bandage pressures are adequately maintained for the whole time during which bandages are worn.

We thank Mr G S Makin and Mr O W Kingdon for their encouragement and advice. This study was supported by a grant from Messrs Elbeo Limited.

\section{References}

${ }^{1}$ Fegan, W G, Proceedings of the Royal Society of Medicine, 1960, 53, 837. 2 Fentem, P H, Goddard, M, and Gooden, B A, British Medical fournal, 1976, 1, 254.

${ }^{3}$ Fentem, P H, Goddard, M, and Gooden, B A, Fournal of Physiology, in press.

4 Somerville, J J F, et al, British fournal of Surgery, 1974, 61, 979.

${ }^{5}$ Ashton, H, British Medical fournal, 1966, 2, 1427.

\title{
Histocompatibility antigens, autoantibodies, and immunoglobulins in alcoholic liver disease
}

\author{
R J BAILEY, N KRASNER, A L W F EDDLESTON, ROGER WILLIAMS, D E H TEE, \\ DEBORAH DONIACH, L A KENNEDY, J R BATCHELOR
}

British Medical fournal, 1976, 2, 727-729

\section{Summary}

Determination of histocompatibility antigens in 63 patients with alcoholic liver disease showed that HLA-B8

Liver Unit, King's College Hospital, London SE5 8RX

R J BAILEY, MD, FRCP(C), research fellow

N KRASNER, MD, MRCP, research fellow

A L W F EDDLESTON, DM, MRCP, senior lecturer in medicine and consultant physician

ROGER WILLIAMS, MD, FRCP, director and consultant physician

Department of Pathology, King's College Hospital, London SE5 8RX D E H TEE, MB, MRCPATH, immunologist

Department of Immunology, Middlesex Hospital, London W1 DEBORAH DONIACH, MD, FRCP, professor of immunology

Tissue Typing Laboratory, Guy's Hospital, London SE1 L A KENNEDY, technician

McIndoe Memorial Research Unit, Queen Victoria Hospital, East Grinstead, Sussex

J R BATCHELOR, MD, director of research was more prevalent in patients with cirrhosis than in controls, but among those with fatty liver and minimal fibrosis the prevalence of this antigen was normal. Another noticeable difference was the absence of HLAA28 in the cirrhotic group. In the total series of 219 patients the prevalence of antinuclear and smooth muscle antibodies was raised; they were especially prevalent in patients with cirrhosis. Raised serum IgA and IgG concentrations were also common (found in $\mathbf{5 0} \%$ and $\mathbf{3 7} \%$ respectively) and were again significantly associated with cirrhosis. In contrast, serum IgM levels, which were raised in $46 \%$ of cases, were not significantly related to the presence of cirrhosis but correlated significantly with the degree of portacaval shunting.

These results support recent evidence suggesting that immune responses may be implicated in alcohol-induced liver damage, particularly in its progression to cirrhosis.

\section{Introduction}

Although the development of cirrhosis in the alcoholic is directly related to the quantity of alcohol and the time taken to consume it, an individual susceptibility also seems to be present. ${ }^{1}$ This 